\title{
Diverging Fluctuations of the Lyapunov Exponents
}

\author{
Diego Pazó, ${ }^{1, *}$ Juan M. López, ${ }^{1, \dagger}$ and Antonio Politi ${ }^{2, \dagger}$ \\ ${ }^{1}$ Instituto de Física de Cantabria (IFCA), CSIC-Universidad de Cantabria, 39005 Santander, Spain \\ ${ }^{2}$ Institute for Complex Systems and Mathematical Biology and SUPA, University of Aberdeen, \\ Aberdeen AB24 3UE, United Kingdom
}

(Received 9 May 2016; published 14 July 2016)

\begin{abstract}
We show that in generic one-dimensional Hamiltonian lattices the diffusion coefficient of the maximum Lyapunov exponent diverges in the thermodynamic limit. We trace this back to the long-range correlations associated with the evolution of the hydrodynamic modes. In the case of normal heat transport, the divergence is even stronger, leading to the breakdown of the usual single-function Family-Vicsek scaling ansatz. A similar scenario is expected to arise in the evolution of rough interfaces in the presence of suitably correlated background noise.
\end{abstract}

DOI: 10.1103/PhysRevLett.117.034101

Lyapunov exponents (LEs) are dynamical invariants that provide a detailed characterization of low-dimensional as well as spatiotemporal chaos [1]: they indeed allow estimating the fractal dimension, the Kolmogorov-Sinai entropy, and allow us to ascertain the extensivity of the underlying dynamical regime. LEs are average quantities, defined as the infinite-time limit of the so-called finite-time Lyapunov exponents (FTLEs). Interestingly, also the temporal fluctuations of FTLEs carry important information that is ultimately encoded in yet another invariant: a suitable large deviation function. Fluctuations help to shed light on important phenomena such as intermittency, strange nonchaotic attractors, and stable chaos [1].

In dissipative systems with many degrees of freedom, the fluctuations of the largest FTLE have been investigated in various numerical setups such as a shell model for the energy cascade in turbulence [2], a cellular automaton [3], molecular dynamics simulations [4], coupled-map lattice models $[5,6]$, and a variety of continuous-time models $[7,8]$. In particular, in spatially extended systems like those in Refs. [5,6,8], the dynamics of Lyapunov vectors, i.e., perturbation fields, is formally equivalent to the evolution of rough interfaces in a noisy environment, the LE corresponding to the velocity of the interface $[9,10]$. This relationship is essentially based on the interpretation of the logarithm of the local amplitude of the perturbation with the height $h(x, t)$ of a suitable interface. As a result, the same "physics" can be found in two significantly different contexts. In particular, the universality class of roughening phenomena identified by the Kardar-ParisiZhang (KPZ) equation [11] includes also the perturbation evolution in spatially extended chaotic systems $[9,10,12]$.

In spite of its broadness, the KPZ universality class does not encompass Hamiltonian models $[13,14]$. Preliminary studies revealed different critical properties and generically attributed the anomalous scaling to long-range correlations [13]. Later on, powerful methods for the characterization of large deviations revealed that extreme fluctuations of the FTLE in the classical Fermi-Pasta-Ulam (FPU- $\beta$ ) chain [15] correspond to atypical solutions of solitonlike and chaotic-breather dynamics $[6,16]$. However, it is not clear to what extent they are responsible for the anomalous nonKPZ behavior.

In this Letter we study the diffusion coefficient $D$ of the maximum FTLE in two prototypical Hamiltonian lattices (the FPU- $\beta$ and the $\Phi^{4}$ models). Contrary to what is observed in dissipative dynamics, where $D$ vanishes in the thermodynamic limit, here $D$ diverges. Within the rough-interface context, this behavior means that the velocity of the interface does not self-average. Otherwise said, the infinite-time limit (implied by the definition of the LE) does not commute with the thermodynamic limit. This is yet another example of how subtle the interrelations between these two limits may be (see also the Hamiltonian mean-field model, where an exchange of limits even transforms a vanishing LE into a finite LE [17]; or stable chaos [18], where the noncommutation of the two limits is at the origin of a self-sustained irregular dynamics in linearly stable environments). Here we show that the divergence of the fluctuations originates from the longrange spatiotemporal correlations that are naturally present in Hamiltonian systems because of conservation laws (notably energy conservation). A similar scenario is expected to arise in the evolution of rough interfaces in the presence of suitably correlated background noise.

Our results complement the pioneering work by McNamara and Mareschal [19], who established a connection between hydrodynamics and Lyapunov dynamics, by analyzing the evolution of the Lyapunov vectors associated to small LEs. Here we shed further light, showing that hydrodynamics shapes the most unstable direction as well.

Theory.-Given an infinitesimal perturbation $\boldsymbol{\delta} \boldsymbol{u}(t)$ pointing along the most unstable direction in tangent space 
(the so-called leading Lyapunov vector), we denote its expansion factor over a time $t$ by $e^{\Gamma(t)}$. The ratio $\lambda(t)=\Gamma(t) / t$, the so-called FTLE, is expected to fluctuate because of the heterogeneity of the degree of instability across phase space. The minimal way to gauge the fluctuations of the FTLE is through the variance

$$
\chi^{2}(t)=\left\langle(\Gamma(t)-\langle\lambda\rangle t)^{2}\right\rangle,
$$

where the angular brackets $\langle\cdots\rangle$ denote an average over an ensemble of trajectories, and $\langle\lambda\rangle$ coincides with the LE $\lim _{t \rightarrow \infty} \lambda(t)$ under the assumption that there is only one ergodic component for the energies considered.

The fluctuations of the FTLE are quantified by the diffusion coefficient

$$
D=\lim _{t \rightarrow \infty} \frac{\chi^{2}}{t}
$$

which is itself a dynamical invariant [i.e., independent of the norm type used to compute $\lambda(t)]$. In extended dynamical systems the diffusion coefficient is expected to scale with the system size $L$ as $D \sim L^{-\gamma}[5,8]$, where $\gamma$ is called the wandering exponent.

The scaling behavior of $D$ can be better understood by interpreting the logarithm of the local amplitude of the perturbation $\left|\delta u_{i}\right|$ as the height of a (rough) surface $[9,10]$ : $h_{i}=\ln \left|\delta u_{i}\right|$. Once introduced, the auxiliary field $\phi_{i}(t)=$ $h_{i}(t)-h_{i}(0)$, its spatial average $\bar{\phi}(t)=(1 / L) \sum_{i=1}^{L} \phi_{i}(t)$ corresponds to $\Gamma(t)$ [20], so that the FTLE coincides with average velocity of the interface.

In the theory of roughening processes one observable of great interest is the (squared) width of the interface $W^{2}(t)=\left\langle\overline{\left(\phi_{i}-\bar{\phi}\right)^{2}}\right\rangle$, which, for self-affine interfaces, satisfies the Family-Vicsek scaling ansatz

$$
W^{2}=L^{2 \alpha} \mathcal{F}\left(t / L^{z}\right)
$$

where $\alpha$ and $z$ are the usual roughness and dynamical exponents, respectively, and $\mathcal{F}(u)=$ const for $u \gg 1$. The validity of this relationship in the context of Lyapunov dynamics in extended dissipative dynamical systems has been repeatedly investigated $[9,10,12,21,22]$, showing that the leading Lyapunov vector falls within the universality class of KPZ dynamics [11].

The observable $\chi^{2}$ defined in Eq. (1) is, within the surface framework, given by $\chi^{2}(t)=\left\langle(\bar{\phi}-\langle\bar{\phi}\rangle)^{2}\right\rangle$. For $\chi^{2}$ it is legitimate to invoke again a scaling ansatz [8]

$$
\chi^{2}=L^{2 \alpha} \mathcal{G}\left(t / L^{z}\right)\left(t / L^{z}\right),
$$

where the explicit time dependence has been included to stress the asymptotic linear growth of $\chi^{2}[\mathcal{G}(u)=$ const for $u \gg 1]$. As a result, the wandering exponent is [8]

$$
\gamma=z-2 \alpha,
$$

for any spatial dimension. In the case of chaotic dissipative systems, $\gamma$ is universal. In fact, as the relationship with KPZ dynamics holds $\alpha=1 / 2, z=3 / 2$ in one dimension, so that $\gamma=1 / 2$; analogously, $\gamma \approx 0.839$ in two dimensions [8]. In both cases $\gamma>0$ implies that the fluctuations of the FTLE decrease upon increasing the system size, thereby indicating that the LE self-averages in the thermodynamic limit.

Models.-The only set of systems with spatiotemporal (extensive) chaos where the correspondence with the KPZ class does not apply is the important class of Hamiltonian models [13]. We investigate two popular Hamiltonian lattices: (i) the FPU- $\beta$ model, defined by the evolution equation $\quad \ddot{q}_{i}=F\left(q_{i+1}-q_{i}\right)-F\left(q_{i}-q_{i-1}\right) \quad$ where $F(x)=x+x^{3}$, and by the tangent space dynamics $\ddot{Q}_{i}=$ $m_{i+1}\left(Q_{i+1}-Q_{i}\right)+m_{i}\left(Q_{i-1}-Q_{i}\right)$, where $Q_{i}$ is the infinitesimal variation of $q_{i}$ and $m_{i}=1+3\left(q_{i}-q_{i-1}\right)^{2}$ is the local multiplier; (ii) the $\Phi^{4}$ model, in which case $\ddot{q}_{i}=q_{i+1}-q_{i}+q_{i-1}-q_{i}^{3}$, and $\ddot{Q}_{i}=Q_{i+1}-m_{i} Q_{i}+Q_{i-1}$, where $m_{i}=1+3 q_{i}^{2}$. In both cases the interface height is defined as $h_{i}(t)=\ln \left|\left[Q_{i}^{2}(t)+\dot{Q}_{i}^{2}(t)\right]^{1 / 2}\right|$. Periodic boundary conditions are always assumed and the equations are integrated by using the McLachlan-Atela algorithm [23]. All simulations were carried out with the moderately high energy density $E / L=5$, which is above the strong stochasticity threshold.

The scaling of the diffusion coefficient $D$ with $L$ has been determined by integrating the equations in tangent space and measuring $\chi^{2}$, as defined by Eq. (1), for different system sizes. The results for the $\Phi^{4}$ model are shown in Fig. 1(a) after a proper rescaling to conform to the scaling ansatz, Eq. (4). There we see that the agreement increases upon increasing the system size with clear evidence that $z \approx 2$. As for $\gamma$, it is convincingly negative, but an accurate estimate of the asymptotic value is problematic due to the slow convergence with the system size. The effective wandering exponent $\gamma_{\mathrm{eff}}(L)$, obtained by comparing the data for $L$ with that for $L / 2$, is plotted in the inset in Fig. 1(a). As a result, we can argue that $\gamma \rightarrow \approx-1$.

The results for the FPU- $\beta$ model are plotted in Fig. 1(b). On the one hand, a robust estimate of $z \approx 3 / 2$ is found for the whole set of system sizes considered in our simulations. On the other hand, $\gamma$ varies with $L$ and makes the estimate of the asymptotic value even more troublesome than in the previous case. The analysis of the effective wandering exponent $\gamma_{\text {eff }}(L)$, plotted in the inset in Fig. 1(b), suggests that $\gamma$ is at least more negative than -0.25 . Given the strong finite-size corrections, we have estimated $\alpha$ independently, from the scaling behavior of the structure factor $\Sigma(k)=\left\langle\left|\hat{h}_{k}(t)\right|^{2}\right\rangle$, where $\hat{h}_{k}(t)$ is the Fourier transform of the interface profile at time $t$. The structure factor for $L=32768$, a fairly large lattice, is shown in Fig. 1(c) 

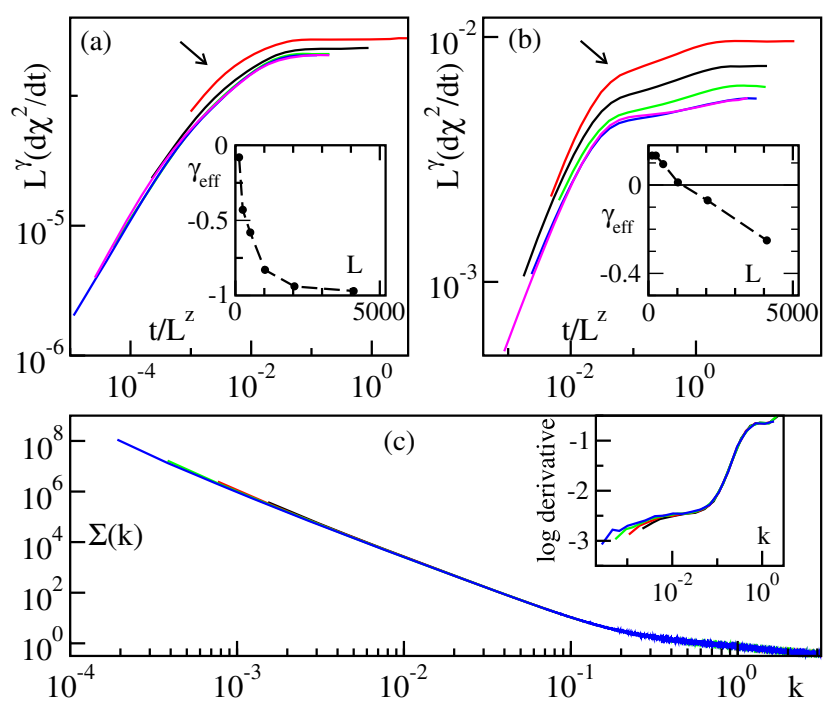

FIG. 1. Rescaled FTLE fluctuations for the $\Phi^{4}$ [panel (a)] and FPU [panel (b)] models, according to the ansatz [Eq. (4)], for sizes $L=256,512,1024,2048,4096$. The derivative $d \chi^{2} / d t$ is considered because of the faster temporal convergence to the asymptotic results. The optimal collapse of the data sets for the larger system sizes is achieved setting $\gamma=-0.97$ and $z=2.15$ in (a) and $\gamma=-0.25$ and $z=1.45$ in (b). The insets show the convergence of $\gamma$, determined by comparing the sizes $L$ and $L / 2$. In panel (c), the structure factor of the Lyapunov-vector surface of the FPU model is plotted for $L=4096,8192,16384$, and 32768 (see black, red, green, and blue lines). The logarithmic derivative is plotted in the inset, adopting the same color coding.

where it appears power-law-like. By virtue of Parseval's theorem one expects $\Sigma(k) \sim k^{-(2 \alpha+1)}$. The inset suggests that there is a very slow convergence to $\alpha=1$ in the thermodynamic limit. This leads us to conjecture the set of exponents for the FPU- $\beta$ model: $\alpha=1, z=3 / 2$, and hence, from Eq. (5), the wandering exponent $\gamma=-1 / 2$. In sum, the relative fluctuations of the FTLE diverge with $L$ in both models, though with different $\gamma$ values.

Given the peculiarities found, we decided to deepen the numerical analysis by looking at the overall issue in a different way. More precisely, we have monitored the ratio between the fluctuations of the FTLE and those of the interface width,

$$
R=\chi^{2} / W^{2}
$$

From Eqs. (3) and (4), this dimensionless observable is expected to be independent of $L$ if plotted versus the rescaled time $u=t / L^{z}$. In Fig. 2(b) we see that this is indeed the case for the FPU- $\beta$ model [there we have assumed $z=1.4$, not far from the value $z=1.45$ estimated from Fig. 1(b)]. There, we also see that $R$ diverges linearly for large $t$, while it grows as $R \sim t^{1 / z}$ at short times, consistently with the scaling Ansätze in Eqs. (3) and (4) [24], see Ref. [8].
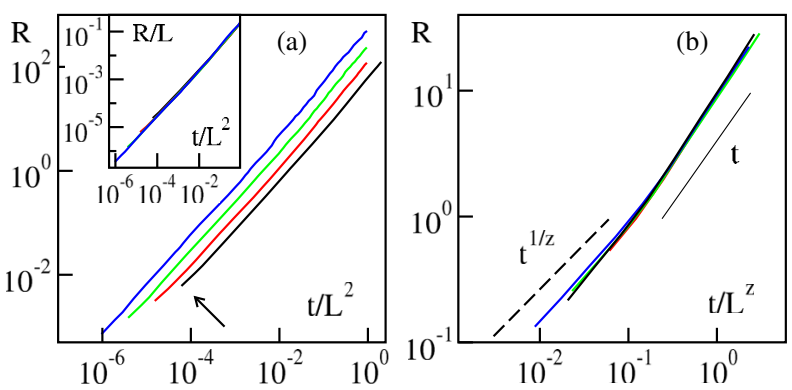

FIG. 2. Evolution of $R$ [see Eq. (6)] in the $\Phi^{4}$ [panel (a)] and FPU [panel (b)] models, for $L=512,1024,2048$, and 4096. $R / L$ is plotted in the inset of panel (a). A good data collapse is achieved for $z=1.4$ for the FPU model.

A completely different scenario is instead found for the $\Phi^{4}$ model. The various curves reported in Fig. 2(a) do not collapse onto one another (neither at short nor at long times). Additionally the time dependence is linear all along the entire range. A nice data collapse is obtained only after rescaling $R$ by $L$ (see the inset). The only way we have found to reconcile this result with the two initial scaling hypotheses is by assuming the existence, for the $\Phi^{4}$ system, of two different $\alpha$ exponents, $\alpha_{\chi}$ and $\alpha_{W}$, in Eqs. (4) and (3), respectively, so that $R \sim L^{2\left(\alpha_{x}-\alpha_{W}\right)}\left(t / L^{z}\right)$. The observed data collapse for the $R$ curves implies $\alpha_{\chi}=\alpha_{W}+1 / 2$. An independent study of the scaling of $W^{2}$ points to $\alpha_{W}=1$ in the thermodynamic limit, whereupon $\alpha_{\chi}=3 / 2$. As a result, the relationship [Eq. (5)] is still valid, once the proper $\alpha$ is being invoked: $\gamma=z-2 \alpha_{\chi}$. This yields $\gamma=-1$, in agreement with the direct simulations in Fig. 1(a).

Stochastic model.-The study of the two Hamiltonian models has revealed a diverging diffusion coefficient of the FTLE fluctuations as well as two significantly different scaling scenarios. Recent progress in the thermodynamic properties of oscillator chains has shown that the two models belong to different universality classes: Fourier law is satisfied in the $\Phi^{4}$ model, while a divergence of transport coefficients is found in the FPU- $\beta$ system [25]. More precisely, the hydrodynamic behavior of $\Phi^{4}$ is a pure diffusion and therefore characterized by $z=2$, while the scenario is more complex in the case of FPU-type models, where $z$ depends on the symmetry of the interactions (see Ref. [26]). Our results show that such a difference manifests itself also in the context of Lyapunov dynamics.

In order to test to what extent the tangent-space dynamics is determined by the correlation properties of the local multipliers $m_{i}(t)$, we have studied the simple model

$$
\delta u_{i}(t+1)=m_{i}(t)\left[\delta u_{i-1}(t)+\delta u_{i}(t)+\delta u_{i+1}(t)\right]
$$

where time is discrete and $m_{i}(t)$ is a stochastic term. It corresponds to the tangent space evolution of a generic coupled-map lattice. If $m_{i}(t)$ is $\delta$ correlated both in space and time, the dynamics of $h_{i}=\ln \left|\delta u_{i}\right|$ belongs to the KPZ 
universality class [9]. In contrast, for the $\Phi^{4}$ model we find that the spectral density of the multipliers is

$$
\left\langle\left|\hat{m}_{k}(\omega)\right|^{2}\right\rangle=\frac{A k^{2}}{B k^{4}+\omega^{2}},
$$

up to some finite-size corrections (data not shown). The form of the hydrodynamic fluctuations given by Eq. (8) corresponds to diffusive transport [27]. Indeed, energy fluctuations relax diffusively in the $\Phi^{4}$ model [25] and this is also expected for other observables like the local multipliers $m_{i}(t)$. In Ref. [28], a simple recipe to generate a stochastic process $m_{i}$ characterized by the spectral density in Eq. (8) was proposed. Given a positive-defined field $m_{i}(t)$, a pair of neighboring sites $i, i+1$ is randomly selected and the conserved quantity $E_{i}=m_{i}(t)+m_{i+1}(t)$ randomly redistributed over the two sites with a uniform probability density in $\left[0, E_{i}\right]$ (so that detailed balance is satisfied). A time unit corresponds to the performance of $L$ random moves.

The scaling behavior of $\chi^{2}$ for the model Eq. (7) is reported in Fig. 3(a) (simulations have been performed for an average $\bar{E}=\sum_{i} E_{i} / L$ equal to 2 ), where we see a scenario quite similar to that of the $\Phi^{4}$ model with a $\gamma$ value close to -1 . The close correspondence is further strengthened by the analysis of $R$ displayed in Fig. 3(b), which confirms that the additional rescaling $R \rightarrow R / L$ is needed to ensure a good data collapse.

Unfortunately, a simple recipe to generate a stochastic process with the correlations expected for the FPU model is not available. We nevertheless believe that there is compelling evidence that the anomalous divergence of the diffusion coefficient emerges from the space-time correlation of the multipliers in tangent space, or, equivalently, of the noise in the rough-interface picture. It is interesting to notice that the anomaly is stronger (i.e., $|\gamma|$ is larger) in the $\Phi^{4}$ model which, thermodynamically, is known to be characterized by "normal" (finite) thermal conductivity. The reason for this seemingly odd conclusion is that the origin of the anomalous Lyapunov dynamics resides in the hydrodynamic behavior of the multipliers: the normal diffusion observed in the $\Phi^{4}$ model is slower than the "anomalous" superdiffusion arising in the FPU- $\beta$ context.

Large-deviation theory.-In order to fully appreciate the role of FTLE fluctuations, it is convenient to introduce the probability $P(\lambda, t, L)$ to observe $\lambda$ over a time $t$ in a system of size $L$. The theory of large deviations predicts that [29-31] (see also Ref. [1])

$$
P(\lambda, t, L) \sim e^{-t S(\lambda, L)},
$$

where the entropy $S(\lambda, L)$ is a dynamical invariant which has typically a quadratic minimum at $\langle\lambda\rangle$ (the true LE). The diffusion coefficient $D$ is the inverse of the second derivative of $S(\lambda, L)$ in $\lambda$ at $\lambda=\langle\lambda\rangle$ (see, e.g., Ref. [5]).
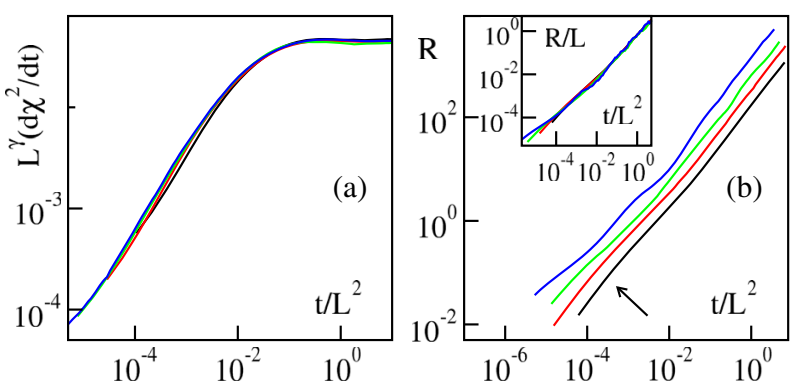

FIG. 3. (a) Rescaled FTLE fluctuations for the stochastic model in Eq. (7), according to the ansatz [Eq. (4)], for $L=256,512$, 1024, and $2048(\gamma=-1)$. (b) The ratio $R$ plotted for the same model and system sizes. The rescaled ratio $R / L$ is plotted in the inset.

Equivalently, one can look at the problem in terms of the generalized LEs $\mathcal{L}(q)$, defined from the growth rate of $q$ th order moments $[32,33]$

$$
\mathcal{L}(q, L)=q^{-1} \lim _{t \rightarrow \infty} t^{-1} \ln \left\langle\|\delta \boldsymbol{u}\|^{q}\right\rangle .
$$

The two representations are connected via a Legendre transform [1]. Since, to lowest order in $q$ [32],

$$
\mathcal{L}(q, L)=\langle\lambda\rangle+q D(L) / 2+O\left(q^{2}\right),
$$

a divergence of $D$ with $L$ implies the existence of a singularity of $d \mathcal{L}(q) / d q$ at $q=0$. Preliminary computations of higher-order cumulants suggest that the divergence is exclusive of the linear term in Eq. (11) due to the presence of nonanalytic terms in the thermodynamic limit of $\mathcal{L}(q, L)$. More refined computational efforts are required to clarify this point.

Conclusions.-We have shown that in Hamiltonian models the variance of the maximal FTLE diverges in the thermodynamic limit. This follows from the slow, hydrodynamic fluctuations that affect the local multipliers. Given the universality classes identified while studying heat conductivity [25], a similar scenario is expected for the Lyapunov fluctuations. In particular, different scaling exponents are expected in the asymmetric FPU- $\alpha$ model. Interestingly, the divergence is stronger and qualitatively different in models exhibiting normal transport such as the $\Phi^{4}$ model. In that case, the structure of the Lyapunov vector surface is not self-affine (i.e., based on a single FamilyVicsek scaling ansatz): two different $\alpha$ exponents must be introduced to describe the growth of the interface width and sample-to-sample fluctuations, respectively. In consonance, the structure factor $\Sigma(k)$ exhibits increasing fluctuations at low $k$. Altogether, we expect the divergence of $D$ to carry over to two- and three-dimensional setups, where normal diffusion is even more universal. At low temperatures the hydrodynamic behavior competes with the intrinsic slowness of the dynamics itself: whether this can lead to further 
anomalies is unknown. Also, we may conjecture the existence of dissipative systems subject to certain conservation laws that may lead to diverging FTLE fluctuations.

In the context of roughening processes, the diverging fluctuations reported here would manifest as a divergence of the fluctuations of the interface velocity. In the past, various noises with a slow decay of either spatial or temporal correlations have been studied and different scaling exponents introduced to characterize the interface evolution [34] (see also Ref. [35]). However, to our knowledge no systematic investigation of combined spatiotemporal correlations has been carried out. This type of combined correlations seems to be essential for the scenario discussed in this Letter to occur.

Finally, regarding the physical meaning of a diverging diffusion coefficient $D$, note this does not imply the violation of the central limit theorem, as for any finite system size $D$ remains finite and thereby the LE well defined. Nevertheless, intermittent phenomena in tangentspace dynamics become increasingly important in the thermodynamic limit. How large are the deviations from a Gaussian approximation is not, however, clear: a complete study of large deviations is required.

D. P. acknowledges support by MINECO (Spain) under a Ramón y Cajal fellowship. We acknowledge support by MINECO (Spain) under Project No. FIS2014-59462-P.

*pazo@ifca.unican.es

${ }^{\dagger}$ lopez@ifca.unican.es

*a.politi@abdn.ac.uk

[1] A. Pikovsky and A. Politi, Lyapunov Exponents, 1st ed. (Cambridge University Press, Cambridge, England, 2016).

[2] A. Crisanti, M. H. Jensen, A. Vulpiani, and G. Paladin, Phys. Rev. Lett. 70, 166 (1993).

[3] A. Crisanti, M. H. Jensen, A. Vulpiani, and G. Paladin, Phys. Rev. A 46, R7363 (1992).

[4] J. R. Green, A. B. Costa, B. A. Grzybowski, and I. Szleifer, Proc. Natl. Acad. Sci. U.S.A. 110, 16339 (2013).

[5] P. V. Kuptsov and A. Politi, Phys. Rev. Lett. 107, 114101 (2011).

[6] T. Laffargue, K.-D. N. T. Lam, J. Kurchan, and J. Tailleur, J. Phys. A 46, 254002 (2013).

[7] H. Nakao and A. S. Mikhailov, Chaos 13, 953 (2003).

[8] D. Pazó, J. M. López, and A. Politi, Phys. Rev. E 87, 062909 (2013).

[9] A. S. Pikovsky and J. Kurths, Phys. Rev. E 49, 898 (1994).
[10] A. Pikovsky and A. Politi, Nonlinearity 11, 1049 (1998).

[11] M. Kardar, G. Parisi, and Y.-C. Zhang, Phys. Rev. Lett. 56, 889 (1986).

[12] D. Pazó and J. M. López, Phys. Rev. E 82, 056201 (2010).

[13] A. Pikovsky and A. Politi, Phys. Rev. E 63, 036207 (2001).

[14] M. Romero-Bastida, D. Pazó, J. M. López, and M. A. Rodríguez, Phys. Rev. E 82, 036205 (2010).

[15] E. Fermi, J. Pasta, and S. Ulam, in Collected Papers of Enrico Fermi (University of Chicago Press, Chicago, 1965), p. 978.

[16] J. Tailleur and J. Kurchan, Nat. Phys. 3, 203 (2007).

[17] F. Ginelli, K. A. Takeuchi, H. Chaté, A. Politi, and A. Torcini, Phys. Rev. E 84, 066211 (2011).

[18] A. Politi and A. Torcini, in Nonlinear Dynamics and Chaos: Advances and Perspectives, Understanding Complex Systems (Springer, Berlin, 2010), pp. 103-129.

[19] S. McNamara and M. Mareschal, Phys. Rev. E 64, 051103 (2001).

[20] Once the geometric norm $\|\delta \boldsymbol{u}\|_{0}=\prod_{i=1}^{L}\left|\delta u_{i}\right|^{1 / L} \quad$ is adopted.

[21] I. G. Szendro, D. Pazó, M. A. Rodríguez, and J. M. López, Phys. Rev. E 76, 025202 (2007).

[22] D. Pazó, I. G. Szendro, J. M. López, and M. A. Rodríguez, Phys. Rev. E 78, 016209 (2008).

[23] R. I. McLachlan and P. Atela, Nonlinearity 5, 541 (1992).

[24] For the norm type considered (and defined by the field $\phi$ ) $\mathcal{G}(u \ll 1)=u^{(2 \alpha+1) / z}[8]$, while $\mathcal{F}(u \ll 1)=u^{2 \alpha / z}[35]$.

[25] Thermal Transport in Low Dimensions, edited by S. Lepri, Lecture Notes in Physics Vol. 921 (Springer, Heidelberg, 2016).

[26] H. Spohn, in Thermal Transport in Low Dimensions, edited by S. Lepri (Springer, New York, 2016), Chap. 3, pp. 107-158.

[27] D. Forster, Hydrodynamic Fluctuations, Broken Symmetry, and Correlation Functions, Frontiers in Physics Vol. 47 (W.A. Benjamin, New York, 1990).

[28] C. Kipnis, C. Marchioro, and E. Presutti, J. Stat. Phys. 27, 65 (1982).

[29] H. Touchette, Phys. Rep. 478, 1 (2009).

[30] P. Grassberger, R. Badii, and A. Politi, J. Stat. Phys. 51, 135 (1988).

[31] M. A. Sepúlveda, R. Badii, and E. Pollak, Phys. Rev. Lett. 63, 1226 (1989).

[32] H. Fujisaka, Prog. Theor. Phys. 71, 513 (1984).

[33] R. Benzi, G. Paladin, G. Parisi, and A. Vulpiani, J. Phys. A 18, 2157 (1985).

[34] E. Medina, T. Hwa, M. Kardar, and Y.-C. Zhang, Phys. Rev. A 39, 3053 (1989).

[35] A.-L. Barabási and H. E. Stanley, Fractal Concepts in Surface Growth (Cambridge University Press, Cambridge, England 1995). 